\title{
123. Über die Nitrierung von Nitrochinolin-N-oxyden.
}

\author{
Von Masawo Ishikawa. \\ Pharmaz. Institut d. Kaiserl. Universität, Tokyo. \\ (Comm. by Y. AsAhINA, M.I.A., Oct. 12, 1944.)
}

Früher haben Ochiai und Ishikawa beim Erhitzen von Chinolin$\mathrm{N}$-oxyd mit Kaliumnitrat in conz. $\mathrm{H}_{2} \mathrm{SO}_{4}$-Lösung 4-Nitroderivat in guter Ausbeute erhalten. Nebenbei entstanden 8- und 5-Nitroderivat in kleiner Menge ${ }^{1)}$. In Fortsetzung der Versuche habe ich nun 4-, 6bzw. 8-Nitrochinolin-N-oxyd weiter nitriert. Die Bedingungen und die Produkte der Reaktion wurden unten tabellarisch zusammengestellt.

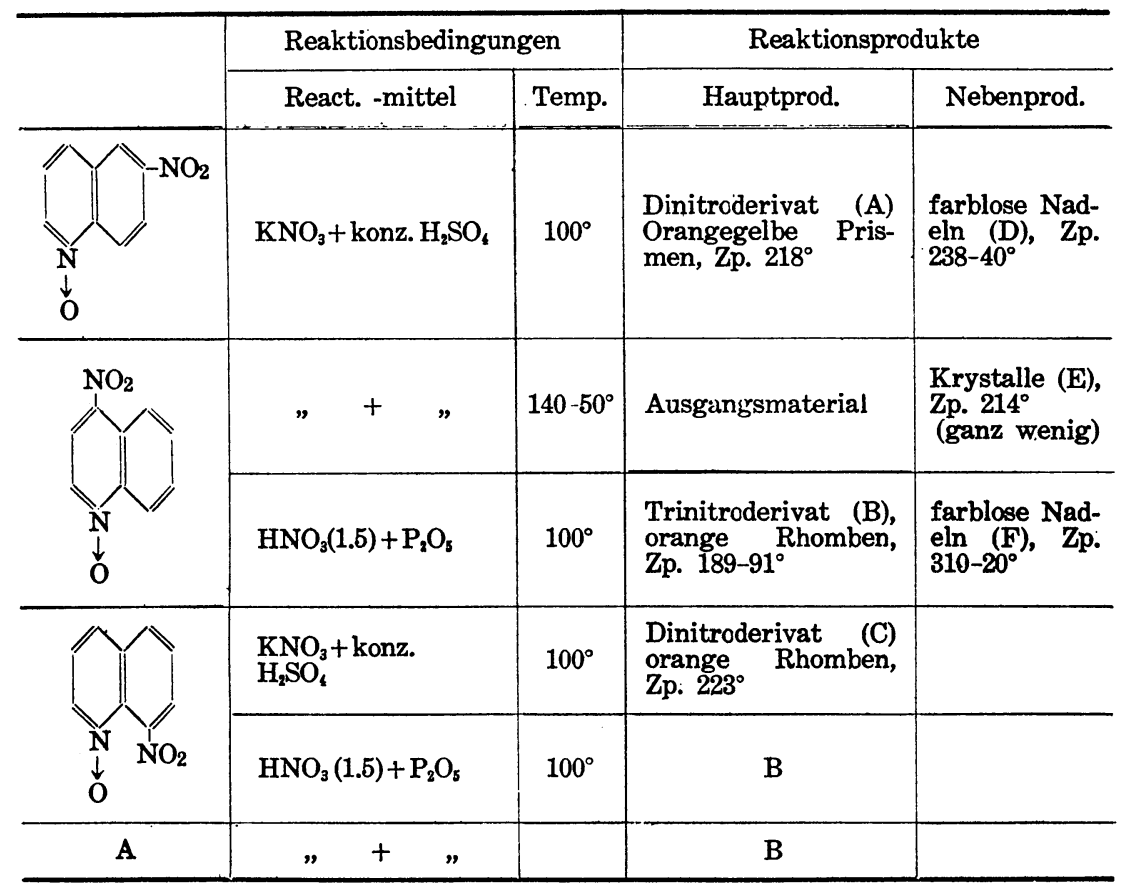

Die Zusammensetzung von $\mathrm{D}, \mathrm{E}$ bzw. $\mathrm{F}$ in der Tabelle wurde noch nicht sicher gestellt.

Das Trinitroderivat B entsteht sowohl aus 4- bzw. 8-Nitrochinolin$\mathrm{N}$-oxyd wie aus $\mathrm{A}$, das Nitroderivat von 6 -Nitrochinolin-N-oxyd, durch analoge Nitrierung. So muss seine Konstitution 4, 6, 8-Trinitrochinolin$\mathrm{N}$-oxyd sein: Das einzig mögliches Bedenken, dass es 4, 6, 8-Trinitrocarbostyril sein könnte wurde beseitigt, indem 4-Nitrochinolin-N-oxyd beim analogen Erhitzen mit Phosphorpentoxyd in konz. $\mathrm{H}_{2} \mathrm{SO}_{4}$-Lösung ganz unverändert bleibt.

Das Dinitroderivat A bzw. C wurde als 4, 6- bzw. 4, 8-Dinitro-

1) E. Ochiai u. M. Ishikawa: J. pharm. Soc., Japan, 63, 280. 
chinolin-N-oxyd festgestellt, weil die beide gegen einander verschieden sind und zwar bei weiterer Nitrierung dasselbe 4, 6, 8-Trinitroderivat geben.

Aus diesen Ergebnissen ersieht man folgendes.

1. Beim unsubstituierten bzw. in der Benzolhälfte nitrierten chinolin- $\mathrm{N}$-oxyd ist die $\mathrm{C}_{4}$-Stellung der Pyridinhälfte am leichtestens nitrierbar.

2. Die Nitrogruppe in der $\mathrm{C}_{4}$-stellung hindert die weitere Nitrierung der Pyridinhälfte. Die Nitrogruppe tritt dann wahrscheinlich zuerst in $\mathrm{C}_{8^{-}}$und dann in $\mathrm{C}_{6}$-Stellung, $d . h$. in der $\mathrm{m}$-Stellung der Benzolhälfte, ein.

Die Reaktionsverlauf kann man somit wie folgt veranschaulichen.<smiles>CCOc1ccc([N+](=O)[O-])c2c(-c3cc(C)c([N+](=O)[O-])c4cc([N+](=O)[O-])c([N+](=O)[O-])cc34)c([N+](=O)[O-])ccc12</smiles>

Es wurde schon bemerkt, dass 4-Nitrochinolin-N-oxyd beim Erwärmen mit Phosphoroxychlorid 2, 4-Dichlorchinolin gibt ${ }^{1}$. Die Nitrogruppe wurde also mit Chlorgruppe ersetzt unter gleichzeitiger UmSetzung der N-Oxydgruppe. Nun wurde es weiter bestätigt, dass die Nitrogruppe in $\mathrm{C}_{4}$-Stellung auch bei den Polynitroderivaten besonders sich verhält.

4, 6-Dinitrochinolin-N-oxyd gibt beim Erwärmen mit Phosphoroxychlorid ein Mononitrodichlorchinolin. Es bildet farblose Nadeln vom Schmp. 192-94 und gibt bei der Reduktion mit Zinnchlorür und Salzsäure das entsprechende Aminodichlorchinolin (gelbe Nadeln vom Schmp. $214^{\circ}$ ), welches bei der katalytischen Reduktion mit Pd-Kohle in HClLösung 6-Aminochinolin vom Schmp. $112-14^{\circ}$ gibt. Hieraus folgt, dass $\mathrm{C}_{4}$-Nitrogruppe von 4, 6-Dinitrochinolin-N-oxyd beim Behandeln mit Phosphoroxychlorid mit Chlorgruppe unter gleichzeitiger. Umsetzung der N-Oxydgruppe ersetzt und 6-Nitro-2, 4-dichlorchinolin entsteht.

4, 6, 8-Trinitrochinolin-N-oxyd gibt dabei ein Dinitrodichlorchinolin als farblose Blättern vom Zp. 209-10 ${ }^{\circ}$, welches sich als 6, 8-Dinitro-2, 4-dichlorchinolin vermuten lässt. Es gibt bei der Reduktion leicht verschmierendes produkt, sodass seine Konstitution noch nicht einwandfrei nachgewiesen wurde. 


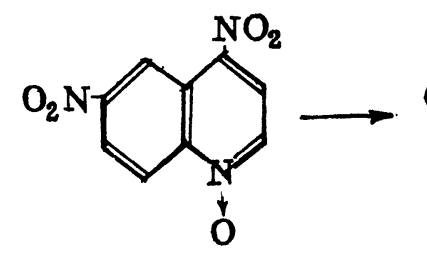<smiles>[CH]c1cc(Cl)c2c(Cl)cc(C)nc2c1</smiles><smiles>Nc1ccc(Cl)cc1Cl</smiles><smiles>O=[N+]([O-])[O-]</smiles><smiles></smiles><smiles>Nc1ccc2ncccc2c1</smiles>

Beschreibung der Versuche. 6-Nitrochinolin- $N$-oxyd.

$4 \mathrm{~g}$ 6-Nitrochinolin wurden in $150 \mathrm{ccm}$ Benzol gelöst, eine aetherische Lösung von Phthalmonopersäure mit $0.6 \mathrm{~g}$ aktivem Sauerstoff hinzugefügt und stehen gelassen. Nach $24 \mathrm{Stdn}$. wurden die ausgeschiedene Krystalle mit Soda zersetzt und aus Aceton umgelöst. Gelbe Prismen vom Schmp. 221 . Ausbeute 2.95g. (67.5\% d. Th.). $3.210 \mathrm{mg}$ Sbst. : $0.398 \mathrm{~cm} \mathrm{~N} \mathrm{~N}_{2}\left(10.5^{\circ}, 766 \mathrm{~mm}\right) ; 3.510 \mathrm{mg}$ Sbst. :

\section{$7.255 \mathrm{mg} \mathrm{CO}, 1.040 \mathrm{mg} \mathrm{H}_{2} \mathrm{O}$. \\ $\mathrm{C}_{3} \mathrm{H}_{6} \mathrm{~N}_{2} \mathrm{O}_{3}$ Ber. $\quad$ C 56.8, $\quad \mathrm{H} 3.2, \quad \mathrm{~N}$ 14.7. \\ Gef. C 56.37, H 3.31, $\mathrm{N}^{-}$15.05.}

Nitrierung von 6-Nitrochinolin-N-oxyd. (4, 6-Dinitrochinolin-N-oxyd)

$1.5 \mathrm{~g}$ 6-Nitrochinolin-N-oxyd wurden in einer Lösung von $2 \mathrm{~g}$ $94 \%$-Schwefelsäure und $4 \mathrm{~g} 10 \%$-rauch. Schwefelsäure gelöst, $1.2 \mathrm{~g}$ Salpeter versetzt und auf dem Wasserbade 21 Stdn. erhitzt. Das Reaktionsgemisch wurde mit $20 \mathrm{ccm}$ Eis zersetzt und die ausgeschiedene Krystalle $(1.7 \mathrm{~g})$ aus Aceton nmkrystallisiert. Orangegelbe Prismen vom $\mathrm{Zp.} 218^{\circ}$. Ausbeute $0.5 \mathrm{~g}$.

$3.340 \mathrm{mg}$ Sbst. : $0.498 \mathrm{~cm} \mathrm{~N} \mathrm{c}_{2}\left(14.5^{\circ}, 765 \mathrm{~mm}\right) ; 3.550 \mathrm{mg}$ Sbst. : $6.030 \mathrm{mg}$ $\mathrm{CO}_{2}, 0.790 \mathrm{mg} \mathrm{H}_{2} \mathrm{O}$.

$$
\begin{array}{lllll}
\mathrm{C}_{9} \mathrm{H}_{5} \mathrm{~N}_{8} \mathrm{O}_{5} & \text { Ber. } & \text { C } 46.0, & \text { H 2.1, } & \text { N 17.9. } \\
& \text { Gef. } & \text { C 46.33, } & \text { H 2.49, } & \text { N 17.82. }
\end{array}
$$

Aus der Mutterlauge der Aceton-Lösung wurden $0.05 \mathrm{~g}$ farblosen Nadeln vom Zp. $238-40^{\circ}$ erhalten. Es ist in Natronlauge löslich mit roter Farbe.

$3.205 \mathrm{mg}$ Sbst.: $5.320 \mathrm{mg} \mathrm{CO}, 1.020 \mathrm{mg} \mathrm{H}_{2} \mathrm{O} ; 3.295 \mathrm{mg}$ Sbst.: $0.294 \mathrm{ccm}$ $\mathrm{N}_{2}\left(14.5^{\circ}, 763 \mathrm{~mm}\right)$.

$\mathrm{C}_{9} \mathrm{H}_{8} \mathrm{~N}_{2} \mathrm{O}_{6} \quad$ Ber. $\quad$ C 45.0, H 3.3, $\quad \mathrm{N}$ 11.7.

Gef. C 45.27, H 3.56, N 10.63 .

Nitrierung von 8-Nitrochinolin- $N$-oxyd. (4, 8-Dinitrochinolin- $N$-oxyd)

$0.5 \mathrm{~g}$ 8-Nitrochinolin-N-oxyd, $2 \mathrm{~g} .94 \% \cdot$ Schwefelsäure, $1 \mathrm{~g}$ rauch. Schwefelsäure $\left(\mathrm{SO}_{3}: 50 \%\right)$ und $0.67 \mathrm{~g}$ Salpeter wurden analogerweise 
wie oben in Einwirkung gebracht und behandelt. Orange Rhomben aus Aceton-Methanol. Z Z $223^{\circ}$

$3.325 \mathrm{mg}$ Sbst. $: 5.725 \mathrm{mg} \quad \mathrm{CO}_{2}, \quad 0.750 \mathrm{mg} \quad \mathrm{H}_{2} \mathrm{O} . \quad 3.160 \mathrm{mg}$ Sbst. : $0.464 \mathrm{ccm} \mathrm{N}_{2}\left(11.5^{\circ}, 761 \mathrm{~mm}\right)$

$\mathrm{C}_{9} \mathrm{H}_{5} \mathrm{~N}_{3} \mathrm{O}_{5}$ Ber. C 46.0, H 2.1, N 17.9, Gef. C 46.96, H 2.52, N 17.64.

Nitrierung von 4-Nitrochinolin- $N$-oxyd.

$0.8 \mathrm{~g}$ 4-Nitrochinolin-N-oxyd wurde $\mathrm{I}$ in einer Lösung von $6 \mathrm{~g} 94 \%$ Schwefelsäure und $2 \mathrm{~g}$ rauch. Schwefelsäure $\left(\mathrm{SO}_{3}: 10 \%\right)$ mit $0.6 \mathrm{~g}$ Salpeter 21 Stdn. auf $140-50^{\circ}$ - erhitzt und mit $20 \mathrm{ccm}$ Eis zersetzt. Die ausgeschiedene Krystalle wurden aus Aceton umgelöst. Gelbe Blättern vom Schmp. $154^{\circ}$. Ausbeute $0.5 \mathrm{~g}$. Eine Mischprobe mit 4Nitrochinolin-N-oxyd schmolz bei $154^{\circ}$.

Als in Aceton schwer lösliche Teil wurde eine Spur von Krystallen vom $\mathrm{Zp}$. $214^{\circ}$ erhalten.

\section{4, 6, 8-Trinitrochinolin- $N$-oxyd.}

1. $\quad 0.4 \mathrm{~g}$ 4-Nitrochinolin-N-oxyd wurden auf einem Gemisch von $0.4 \mathrm{ccm}$ konz. Salpetersäure $(\mathrm{d}=1.5)$ und $0.2 \mathrm{~g}$ Phosphorpentoxyd versetzt und bis Aufhören der Entwicklung von nitrosen Gasen auf dem Wasserbade (ca. 14 Stdn.) erwärmt. Das Reaktionsgemisch wurde mit $20 \mathrm{ccm}$ Eis zersetzt und die ausgeschiedene Krystalle $(0.3 \mathrm{~g})$ aus Aceton umgelöst. Von dem in Aceton leichter löslichen Teil wurden orangegelbe Rhomben vom $\mathrm{Zp}$. $189-91^{\circ}$ erhalten.

$3.095 \mathrm{mg}$ Sbst.: $4.340 \mathrm{mg} \mathrm{CO}_{2}, 0.420 \mathrm{mg} \mathrm{H}_{2} \mathrm{O} ; 2.920 \mathrm{mg}$ Sbst. : $0.495 \mathrm{ccm} \mathrm{N}_{2}\left(22^{\circ}, 759 \mathrm{~mm}\right)$

$\mathrm{C}_{9} \mathrm{H}_{4} \mathrm{~N}_{4} \mathrm{O}_{7} \quad$ Ber. $\quad$ C 38.6, $\quad \mathrm{H}$ 1.4, $\quad \mathrm{N} 20.0$.

Gef. C 38.24, H 1.51, N 19.60 .

Von dem in Aceton schwerer löslichen Teil wurden farblose Nadeln vom $\mathrm{Zp}$. $310-20^{\circ}$ in kleiner Menge erhalten.

$3.175 \mathrm{mg}$ Sbst. : $6.290 \mathrm{mg} \mathrm{CO}, 0.830 \mathrm{mg} \quad \mathrm{H}_{2} \mathrm{O}$; $3.320 \mathrm{mg}$ Sbst. : $0.376 \mathrm{~cm} \mathrm{~N} \mathrm{~N}_{2}\left(22^{\circ}, 759 \mathrm{~mm}\right)$

Gef. C 54.03, H 2.92, N 13.12.

2. $\quad 0.5 \mathrm{~g}$ 8-Nitrochinolin-N-oxyd, 0,5 g Phosphorpentoxyd, und $1.5 \mathrm{ccm}$ rauch Salpetersäure wurden wie bei 1 in Einwirkung gebracht und analogerweise behandelt. Gelbe Rhomben vom $\mathrm{Zp}$. $190^{\circ}$. Ausbeute $0.5 \mathrm{~g}$. Eine Mischprobe mit dem Trinitroderivat aus 4-Nitrochinolin- $\mathrm{N}$-oxyd zeigte keine Depression.

3. $0.5 \mathrm{~g}$ 4,6-Dinitrochinolin-N-oxyd (das Nitrierungsprodukt von 6-Nitrochinolin-N-oxyd), $0.5 \mathrm{~g}$ Phosphorpentoxyd und $1.5 \mathrm{ccm}$ rauch. Salpetersäure wurden wie oben behandelt. Gelbe Rhomben vom $\mathrm{Zp}$. 186-90 . Ausbeute $0.2 \mathrm{~g}$. Eine Mischprobe mit dem Trinitroderivat aus 4-Nitrochinolin-N-oxyd zeigte keine Depression.

6-Nitro-2, 4-dichlorchinolin- $N$-oxyd.

$0.2 \mathrm{~g} \mathrm{4}$, 6-Dinitrochinolin-N-oxyd wurden mit $2 \mathrm{~g}$ Phosphoroxychlorid auf dem Wasserbade 2 Stdn. erwärmt, wobei eine lebhafte Entwicklung von nitrosen Gasen (blaue Färbung des Jodstärkepapiers) bemerkt. Das Reaktionsmischung wurde mit $20 \mathrm{ccm}$ Eis zersetzt und 
die ausgeschiedene Krystalle ausgeäthert. Feine Nadeln aus Äther. Schmp. $192-94^{\circ}$. Ausbeute 0.18 g. $3.660 \mathrm{mg}$ Sbst.: $5.920 \mathrm{mg} \mathrm{CO}$, $0.565 \mathrm{mg} \mathrm{H}_{2} \mathrm{O} ; 3.105 \mathrm{mg}$ Sbst. : $0.298 \mathrm{~cm} \mathrm{~N} \mathrm{~N}_{2}\left(13.5^{\circ}, 756 \mathrm{~mm}\right)$.

$\mathrm{C}_{9} \mathrm{H}_{4} \mathrm{O}_{2} \mathrm{~N}_{2} \mathrm{Cl}_{2}$ Ber. C 44.4, H 1.6, N 11.5. Gef. C 44.11, H 1.73, N 11.37.

$10 \mathrm{~g}$. wasserfreie Zinnchlorür wurden auf $50 \mathrm{ccm}$ getrockneten Äther suspendiert und mit trocknem Salzsäuregas bis 'klare Lösung eingeleitet. $\mathrm{Zu}$ dieser Lösung wurden $0.4 \mathrm{~g}$ oben erhaltene Probe (Smp. 192 94") versetzt, die gleich danach ausgeschiedene Krystalle mit Natronlauge zersetzt und ausgeäthert. Gelbe Nadeln aus Äther. Schmp. $214^{\circ}$. Ausbeute $0.2 \mathrm{~g}$.

$3.350 \mathrm{mg}$ Sbst. : $6.285 \mathrm{mg} \mathrm{CO}, 0: 850 \mathrm{mg} \mathrm{H}_{2} \mathrm{O} ; 3.850 \mathrm{mg}$ Sbst. : $0.422 \mathrm{ccm}$ $\mathrm{N}_{2}\left(15^{\circ}, 762 \mathrm{~mm}\right)$

$\mathrm{C}_{9} \mathrm{H}_{8} \mathrm{~N}_{2} \mathrm{Cl}_{2} \quad$ Ber. $\quad$ C 51.2, $\quad \mathrm{H}$ 2.8, $\quad \mathrm{N} 13.1$.

Gef. C 50.70, H 2.81, N 13.03.

Die gesamte Aminoderivat wurde in $20 \mathrm{ccm} 10 \%$ Salzsäure gelöst, mit Pd-Kohle (aus $0.1 \mathrm{~g}$ Kohle und $10 \mathrm{ccm} 1 \%$ Pd-chlorür-Lösung bereitet) katalytisch reduziert. $\mathrm{H}_{2}$-Aufnahme: ca. $45 \mathrm{ccm}$. Nadeln aus Äther. Schmp. 112-14 ${ }^{\circ}$. Eine Mischprobe-mit 6-Aminochinolin schmolz bei $112-14^{\circ}$.

Dinitro-2, 4-dichlorchinolin.

$0.8 \mathrm{~g}$ Trinitroderivat aus 4-Nitrochinolin-N-oxyd wurden mit $8 \mathrm{ccm}$ Phosphoroxychlorid und $0.5 \mathrm{~g}$ Phosphorpentachlorid $5 \mathrm{Stdn}$. auf dem Wasserbade erwärmt. Das Reaktionsgemisch wurde im Vacuum destilliert und der von Phosphoroxychlorid befreite Rückstand mit Eis zersetzt. Die hierbei ausgeschiedene Krystalle wurden aus Aceton umgelöst. Farblose Rhomben vom $\mathrm{Zp} .209-10^{\circ}$. Ausbeute $0.7 \mathrm{~g}$.

$3.040 \mathrm{mg}$ Sbst.: $4.210 \mathrm{mg} \mathrm{CO}, 0.300 \mathrm{mg} \mathrm{H}_{2} \mathrm{O} ; 3.730 \mathrm{mg}$ Sbst.: $0.465 \mathrm{ccm}$ $\mathrm{N}_{2}\left(22^{\circ}, 753 \mathrm{~mm}\right)$

$\mathrm{C}_{9} \mathrm{H}_{3} \mathrm{~N}_{3} \mathrm{O}_{4} \mathrm{Cl}_{2}$ : Ber. C 37.5, $\mathrm{H}$ 1.01, N 14.6.

Gef. C 37.77, $\mathrm{H}$ 1.10, N 14.29. 